\title{
Ultra-processed food consumption and breast cancer risk
}

\author{
Inge Huybrechts $^{1}$, Isabelle Romieu ${ }^{1}$, Neha Kandpur ${ }^{2}$, Katerina Katsikari ${ }^{1}$, \\ Gabriela Torres-Mejia ${ }^{3}$, Gloria I. Sanchez ${ }^{4}$, Maria Luisa Garmendia ${ }^{5}$, Carolina Porras ${ }^{6}$, \\ Carine Biessy ${ }^{1}$, Marc J. Gunter ${ }^{1}$, Carlos Augusto Monteiro ${ }^{2}$ and Sabina Rinaldi ${ }^{1}$ \\ ${ }^{1}$ International Agency for Research on Cancer, Lyon, France, \\ ${ }^{2}$ Universidade de São Paulo, Sao Paulo, Brazil, \\ ${ }^{3}$ National Institute of Public Health, Cuernavaca, Morelos, Mexico, \\ ${ }^{4}$ University of Antioquia, Medellin, Colombia, \\ ${ }^{5}$ Institute of Nutrition and Food Technology, Universidad de Chile, Santiago de Chile, Chile and \\ ${ }^{6}$ Agencia Costarricense de Investigaciones Biomédicas-Fundación INCIENSA (ACIB-FUNIN), San José, Costa Rica
}

\begin{abstract}
Objectives: The consumption of ultra-processed foods (UPF) has been linked to an increased risk of cancer in western populations, particularly for breast cancer (BC). Although the consumption of UPF is increasing rapidly in Latin American (LA) populations too, there has been no evaluation of its association with BC among LA women, where $20 \%$ of the BC cases present at ages younger than 45 years. We therefore evaluated the role of UPF intake on BC risk in young women participating in the Latin American PRECAMA study.

Methods: The PRECAMA study is an ongoing study coordinated by the International Agency for Research on Cancer (IARC) and involves scientific teams in four LA countries (Chile, Colombia, Costa Rica and Mexico); 406 incident BC cases and population based controls aged 20-45 years are recruited. Trained nurses administered a lifestyle and food frequency questionnaires (FFQ), conducted anthropometric measurements and collected biological samples. Tumour receptor status are determined in a centralized laboratory. UPF consumption was estimated by applying the NOVA classification to the dietary consumption data obtained via the FFQs. The association of the consumption of UPF and $\mathrm{BC}$ was determined using conditional logistic regression analysis adjusting for potential confounding factors.
\end{abstract}

Results: The median age at recruitment was 40 years. The median percentage of calories from UPF was $24 \%\left(10^{\text {th }}\right.$ to $90^{\text {th }}$ percentile: 9 to $43 \%$ ). UPF intake was positively associated with $\mathrm{BC}$ risk (OR for tertiles 2 and 3 in comparison with tertile 1 were 1.84 (95\% CI $1.21-2.81)$ and 1.69 (95\% CI $0.99-2.89)$ respectively). Among the UPF most strongly associated with BC, we identified the intake of industrial bread, packaged sweet and savoury snacks, breakfast cereals, cakes and desserts, and ready-eat/fast food. Comparing the $3^{\text {rd }}$ with the $1^{\text {st }}$ tertile of energy intake from the UPF group "fast food" the OR for BC risk was 1.93 (95\% CI 1.23-3.04).

Discussion: Our results show positive associations between the consumption of UPF and BC risk in young women in LA. Therefore, global actions to decrease UPF intakes are urgently needed to address the cancer burden. In addition, further studies are needed to disentangle mechanisms relating UPF intake and carcinogenic processes in the breast. The European Prospective Investigation into Cancer and Nutrition (EPIC) study, a large-scale cohort including 500,000 participants and 18,814 BC cases will be used to unravel the mechanistic pathways underlying these positive associations between the consumption of UPF and BC risk.

\section{Conflict of Interest}

There is no conflict of interest 